\title{
Improved Bath Sonication Method for Dispersion of Individual Single-Walled Carbon Nanotubes Using New Triphenylene-based Surfactant
}

Tatsuhiro YAMAMOTO ${ }^{1}$, Yuhei MIYAUCHI ${ }^{2}$, Jin MOTOYANAGI ${ }^{3}$, Takanori FUKUSHIMA ${ }^{1,3,4}$, Takuzo AIDA ${ }^{1,3,4}$, Masaru KATO ${ }^{1,5, *}$, and Shigeo MARUYAMA ${ }^{2, *}$

${ }^{1}$ Center for NanoBio Integration, The University of Tokyo, 7-3-1 Hongo, Bunkyo-ku, Tokyo 113-8656, Japan

2 Department of Mechanical Engineering, Graduate School of Engineering, The University of Tokyo, 7-3-1 Hongo, Bunkyo-ku, Tokyo 113-8656, Japan

${ }^{3}$ ERATO-SORST Nanospace Project, Japan Science and Technology Agency, 2-41 Aomi, Koto-ku, Tokyo 135-0064, Japan

${ }^{4}$ Department of Chemistry and Biotechnology, Graduate School of Engineering, The University of Tokyo, 7-3-1 Hongo, Bunkyo-ku, Tokyo 113-8656, Japan

${ }^{5}$ Department of Applied Chemistry, Graduate School of Engineering, The University of Tokyo, 7-3-1 Hongo, Bunkyo-ku, Tokyo 113-8656, Japan

${ }^{*}$ Corresponding authors

e-mail: maruyama@photon.t.u-tokyo.ac.jp

We developed a new surfactant for the dispersion of single-walled carbon nanotubes (SWNTs). The surfactant is an amphiphilic compound with a $\pi$-conjugative triphenylene skeleton. SWNTs were individually dispersed by the aid of the surfactant using a common low-power bath sonicator. Well-dispersed HiPco SWNTs in $\mathrm{D}_{2} \mathrm{O}$ solution were obtained using the surfactant by the repetition of surfactant addition and sonication, followed by overnight sonication. Spectroscopic measurements of the dispersed solution revealed that the SWNTs were dispersed sufficiently to show clear photoluminescence (PL) without ultracentrifugation. Moreover, this method was applied to the dispersion of SWNTs using a typical surfactant, sodium dodecylbenzene sulfate (SDBS). The observed clear PL from SWNTs in the solution obtained without ultracentrifugation suggested that gradual surfactant addition and sonication, followed by overnight sonication, strongly contributed to the efficient dispersion of SWNTs in water.

Keywords: single-walled carbon nanotubes (SWNTs), dispersion agents, optical absorption, bath sonicator, photoluminescence

\section{Introduction}

The development of a simple and versatile method for dispersing carbon nanotubes is essential not only for the utilization of the nanotubes in various fields, but also for the detailed understanding of the physical properties of the nanotubes. In particular, single-walled carbon nanotubes (SWNTs) have attractive electronic properties, since they become metallic or semiconductive depending on chiral indices $(n, m){ }^{1)}$ However, their strong tendency to form bundles (or ropes) due to strong van der Waals interactions prevents their utilization as the ultimate nanomaterial. To disperse SWNTs into solvents, many dispersion reagents, including surfactants, ${ }^{2-5)}$ 
porphyrin, ${ }^{6)}$ polycyclic aromatic hydrocarbons, ${ }^{7,8)}$ DNA, ${ }^{9,10)}$ peptides, ${ }^{11)}$ and polysaccharides, ${ }^{5,12,13)}$ have been reported. Furthermore, the successful dispersion of SWNTs could lead to the diameter and/or chirality-selective separation of individual SWNTs. ${ }^{11,14-16)}$ To achieve effective dispersion, horn-type high-power sonicators are usually applied to a dispersant-containing suspension of SWNTs. However, such a harsh treatment is known to deteriorate the inherent properties of SWNTs, and more moderate methods for SWNT dispersion have been challenged. ${ }^{17,18)}$ Here, we report a new, efficient technique for dispersing SWNTs that yields individual nanotubes under mild sonication conditions using an inexpensive, common bath sonicator. This finding was obtained by the evaluation of a newly designed triphenylene-based surfactant.

\section{Experimental Methods}

\subsection{Materials}

Unless otherwise noted, all commercial reagents and solvents were used as received. Purified HiPco SWNTs were supplied from Rice University. sodium dodecylbenzene sulfate (SDBS) was purchased from Wako Chemicals.

\subsection{Instruments}

Sonication and ultracentrifugation were performed on a Branson model 5510 (180 W, 42 $\mathrm{kHz}$ ) and a Hitachi Koki model Himac CS120GX, equipped with an S100AT6 angle rotor, respectively. Optical absorption spectra were recorded using a Shimadzu model UV-3150 spectrometer. Photoluminescence (PL) spectra were measured using a HORIBA model SPEX Fluorolog-3-11 spectrofluorometer, equipped with a liquid-nitrogen-cooled InGaAs near-IR detector. The excitation and emission spectral slit widths were both $10 \mathrm{~nm}$, and scan steps were $5 \mathrm{~nm}$ on both axes.

\subsection{Synthesis of TP-CO $2 \mathrm{H}$}

A dimethylformamide (DMF) suspension of a mixture of 2,3,6,7,10,11-hexahydroxytriphenylene hydrate (802 mg, $2.47 \mathrm{mmol}$ ), ethyl 11-bromoundecanoate (7.3 g, $25 \mathrm{mmol})$, and $\mathrm{K}_{2} \mathrm{CO}_{3}(4.3 \mathrm{~g}, 31 \mathrm{mmol})$ was refluxed for $15 \mathrm{~h}$ under argon. After being cooled to $25{ }^{\circ} \mathrm{C}$, the formed insoluble fraction thus formed was filtered off and washed with ethyl acetate (AcOEt). The filtrate was evaporated to dryness under reduced pressure. The residue was chromatographed on silica gel with hexane/AcOEt (AcOEt: gradient 10-50\%) as eluents to allow the isolation of TP-CO $\mathrm{CO}_{2}$ Et (Fig. 1(a)) as a waxy pale yellow solid substance (3.3 g, $2.1 \mathrm{mmol}$ ) in $84 \%$ yield. IR $\left(\mathrm{KBr}, \mathrm{cm}^{-1}\right)$ 1174, 1262, 1389, 1438, 1468, 1519, 1617, 1737, 2852, 2924. ${ }^{1} \mathrm{H}$ NMR (CDCl $), \delta(\mathrm{ppm}) 7.81(\mathrm{~s}, 6 \mathrm{H}), 4.20(\mathrm{t}, J=6.4 \mathrm{~Hz}, 12 \mathrm{H}), 4.10(\mathrm{q}, J=7.0 \mathrm{~Hz}, 12 \mathrm{H}), 2.26(\mathrm{t}, J$ = $7.6 \mathrm{~Hz}, 12 \mathrm{H}), 1.94-1.87(\mathrm{~m}, 12 \mathrm{H}), 1.62-1.15(\mathrm{~m}, 24 \mathrm{H}), 1.41-1.25(\mathrm{~m}, 60 \mathrm{H}), 1.23(\mathrm{t}, J=7.0 \mathrm{~Hz}$, 18H). ${ }^{13} \mathrm{C} \mathrm{NMR}\left(\mathrm{CDCl}_{3}\right): \delta$ (ppm) 173.7, 148.9, 123.6, 107.4, 69.7, 60.2, 34.4, 29.7, 29.6, 29.5, 29.4, 29.2, 26.3, 25.1, 14.4. MALDI-TOF (Matrix Assisted Laser Desorption/Ionization-Time of Flight) mass: calculated for $\mathrm{C}_{96} \mathrm{H}_{156} \mathrm{O}_{18}[\mathrm{M}]^{+}: \mathrm{m} / \mathrm{z}=1597.13$; found, 1597.35. EtOH (20 mL) and aqueous $\mathrm{KOH}(2.3 \mathrm{M}, 10 \mathrm{~mL})$ were added to a THF $(20 \mathrm{~mL})$ solution of TP-CO $2 \mathrm{Et}(1.25 \mathrm{~g}, 0.782$ $\mathrm{mmol}$ ), and the mixture was refluxed for $15 \mathrm{~h}$ under argon. The reaction mixture was allowed to cool to $25{ }^{\circ} \mathrm{C}$, acidified with aqueous $\mathrm{HCl}(1 \mathrm{~N})$, and then extracted with $\mathrm{CH}_{2} \mathrm{Cl}_{2}$. The combined organic extract was washed with water, dried over $\mathrm{Na}_{2} \mathrm{SO}_{4}$, and then evaporated to dryness under 
reduced pressure. The residue was recrystallized from $\mathrm{CH}_{3} \mathrm{CN}$ to give $\mathrm{TP}-\mathrm{CO}_{2} \mathrm{H}$ (Fig. 1(a)) as white solid (1.03 g, $0.720 \mathrm{mmol})$ in 92\% yield. IR $\left(\mathrm{KBr}, \mathrm{cm}^{-1}\right)$ 1172, 1263, 1389, 1436, 1468, 1518, 1618, 1707, 2851, 2922. ${ }^{1} \mathrm{H}$ NMR (DMSO- $\left.d_{6}\right): \delta$ (ppm) 11.90 (s, 6H), 7.91 (s, 6H), 4.19 (t, $J=6.1 \mathrm{~Hz}$, $12 \mathrm{H}$ ), $2.16(\mathrm{t}, J=7.3 \mathrm{~Hz}, 12 \mathrm{H}), 1.85-1.73(\mathrm{~m}, 12 \mathrm{H}), 1.55-1.41$ (m, 24H), 1.39-1.22 (m, 60H). ${ }^{13} \mathrm{C} \mathrm{NMR}\left(\mathrm{CDCl}_{3}\right): \delta(\mathrm{ppm})$ 174.2, 148.3, 122.7, 107.0, 68.6, 33.7, 29.1, 28.9, 28.8, 28.6, 25.8, 24.5. MALDI-TOF mass: calculated for $\mathrm{C}_{84} \mathrm{H}_{132} \mathrm{O}_{18}[\mathrm{M}]^{+}: \mathrm{m} / \mathrm{z}=1428.94$; found: 1428.74 .

\subsection{Procedure for Dispersion of SWNTs}

Typically, a $\mathrm{D}_{2} \mathrm{O}$ solution ( $1 \mathrm{~mL}$ ) containing $0.2 \%(\mathrm{w} / \mathrm{v})$ of TP, prepared by the reaction of TP- $\mathrm{CO}_{2} \mathrm{H}$ with an equimolar amount of $\mathrm{NaOH}$, was added to SWNTs in a glass tube (Fig. 2(a)). The resulting suspension was sonicated for $30 \mathrm{~min}$, where the mixture was occasionally stirred. This addition-sonication process was repeated 6 times, and the final composition of SWNTs and TP was 1/12 (w/w). The SWNT dispersion was further sonicated overnight and then ultracentrifuged for $1 \mathrm{~h}$ at $386000 \mathrm{~g}$. The resulting homogeneously dispersed solution was used in the following analysis.

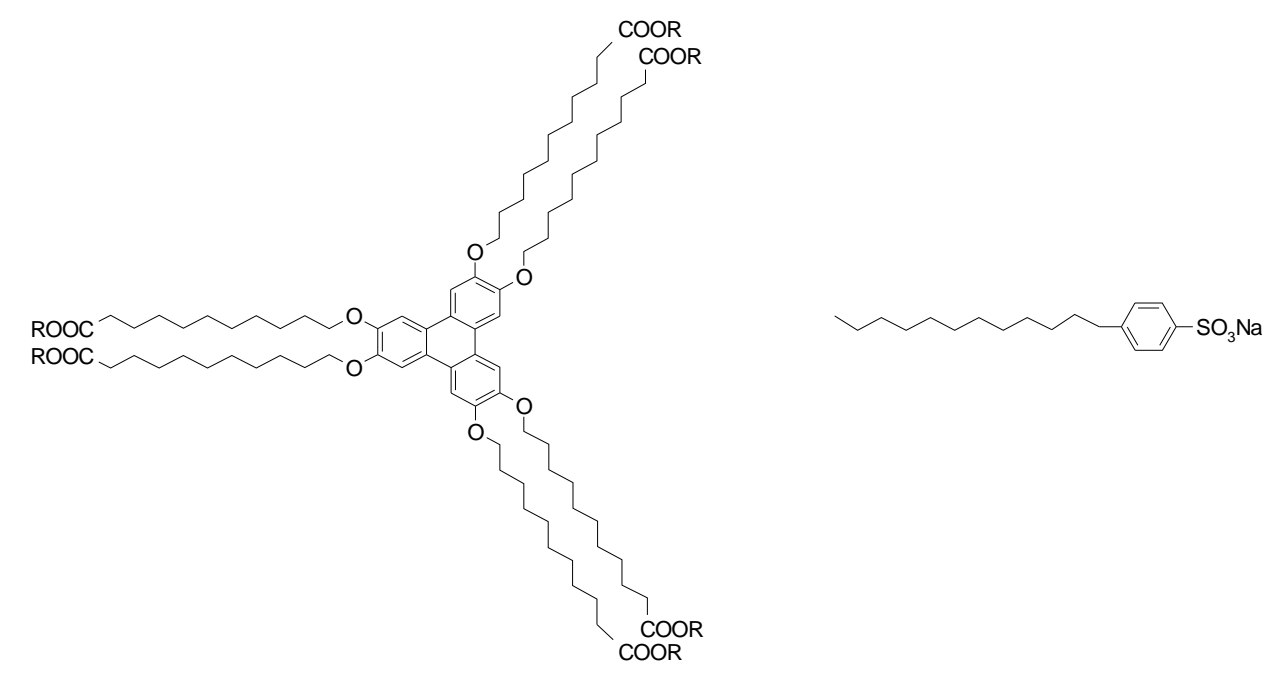

$\mathrm{TP}-\mathrm{CO}_{2} \mathrm{H}, \mathrm{R}=\mathrm{H}$

TP- $-\mathrm{CO}_{2} \mathrm{Et}, \mathrm{R}=\mathrm{CH}_{2} \mathrm{CH}_{3}$

$T P, R=N a$

(a)

(b)

Fig. 1. Schematic structures of (a) triphenylene derivatives and (b) SDBS. 
(a) Stepwise addition

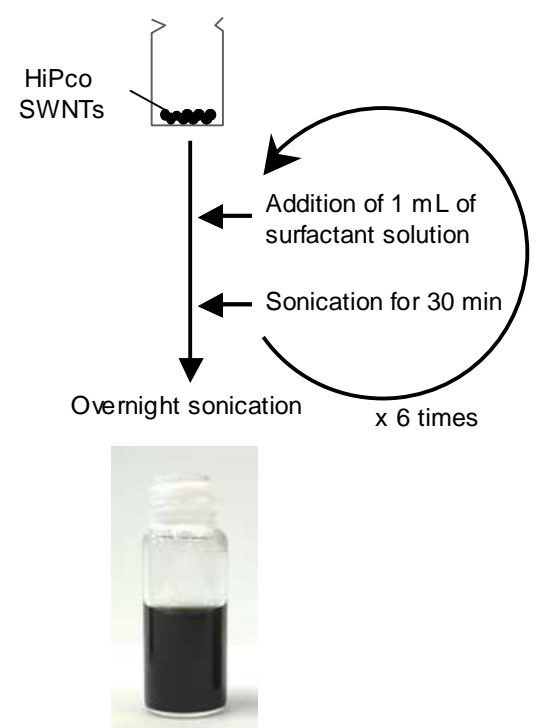

(b) Conventional addition

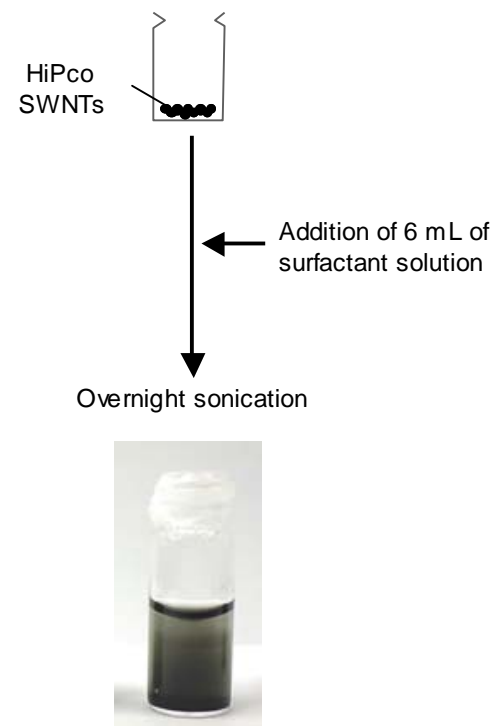

Fig. 2. (a) Procedures for dispersion of SWNTs in water by stepwise addition of aqueous TP solution $(0.2 \%(w / v))$. (b) Comparison of photographs of SWNT dispersions.

\section{Results and Discussion}

Many kinds of amphiphilic aromatic compounds have been used as dispersion reagents for SWNTs in water, because $\pi$-electron-rich groups exhibit strong affinity for SWNTs. ${ }^{7,8)}$ We chose a triphenylene-based surfactant (TP; Fig. 1(a)) for this reason. The surfactant was expected to first bind to SWNTs by the $\pi-\pi$ interaction between the surface of the SWNTs and the triphenylene skeleton of TP, followed by the alkyl chain of TP wrapping around the SWNTs owing to hydrophobic and van der Waals interactions in water.

To obtain SWNT dispersions, we added different volumes of TP solution to the SWNTs. We prepared the mixture solutions of SWNTs and TP with ratios from 1/2 to 1/16 (w/w). Although the mixtures were sonicated overnight using a bath sonicator, SWNTs were not well dispersed. However, we accidentally obtained a well-dispersed SWNT solution by the addition of TP in the stepwise procedure shown in Fig. 2(a). The mixing ratio between SWNTs and TP was the same, but the appearance of this dispersed solution was completely different from those prepared by the procedure shown in Fig. 2(b). This result indicates that the procedure is critical in obtaining dispersions of SWNTs with TP. Figure 3(a) shows a photograph of SWNTs dispersed with TP using the bath sonicator. Few precipitations were observed in the solution stored at room temperature for several weeks.

This stepwise dispersion technique was applied to other surfactants for the dispersion of SWNTs in water in order to evaluate whether the procedure is general. Since SDBS is a typical surfactant commonly used for SWNT dispersion, we chose SDBS for the evaluation of this dispersion procedure (Fig. 2(a)). A well-dispersed solution was obtained with SDBS, and almost no differences were observed between the solution dispersed with TP and that with SDBS after overnight sonication (Figs. 3(a) and 3(c)). This result indicated that SWNTs were also dispersed 
well in water by the stepwise addition technique using SDBS as a surfactant. This dispersion technique is a useful and promising procedure for the dispersion of SWNTs in solution without a strong horn-type sonicator. Figures 3(b) and 3(d) show photographs of the supernatant solution after ultracentrifugation. The solution became transparent because some bundles and impurities were removed by ultracentrifugation. The black color of the supernatant solution with SDBS (Fig. 3(d)) was darker than that of the solution with TP (Fig. 3(b)).

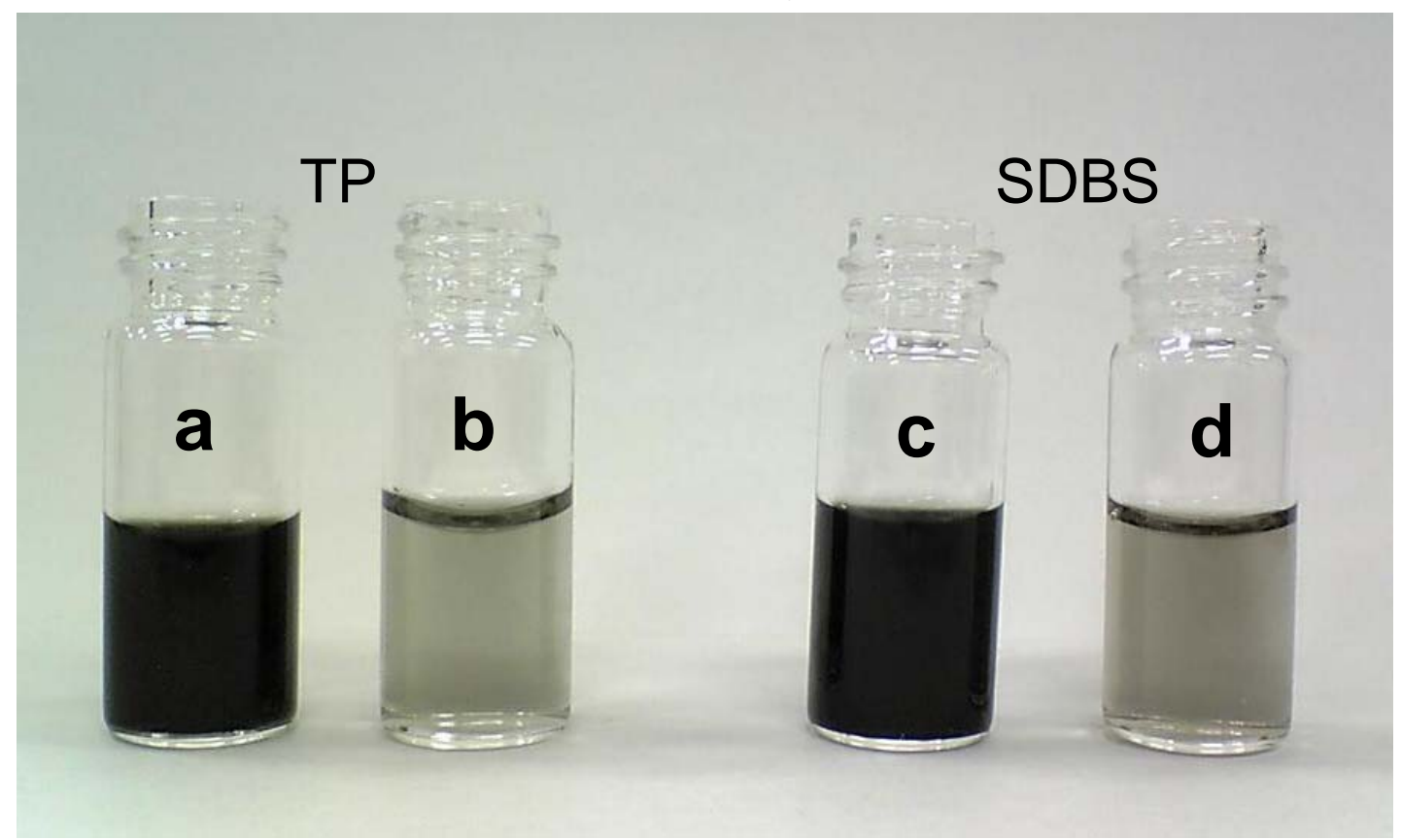

Fig. 3. Photographs of $\mathrm{D}_{2} \mathrm{O}$ dispersions of SWNTs with (a) TP, (b) TP after ultracentrifugation, (c) SDBS, and (d) SDBS after ultracentrifugation.

UV-vis-NIR (ultraviolet-visible-near infrared) absorption and photoluminescence (PL) spectroscopy maps were used in the evaluation of the SWNT solutions. As reported by Bachilo et al., ${ }^{19)}$ characteristic absorption and emission spectra from individual semiconducting SWNTs were observed in the dispersed solution, and these spectra were useful for determining specific nanotube structures, namely, chiral indices $(n, m)$, which describe the tube diameter and SWNT structure. Therefore, we began by measuring UV-vis-NIR spectra of the dispersed solutions.

UV-vis-NIR absorption spectra of $\mathrm{D}_{2} \mathrm{O}$ dispersions of SWNTs with (a) TP, (b) TP after ultracentrifugation, (c) SDBS, and (d) SDBS after ultracentrifugation are shown in Fig. 4. Since the absorbance of the solutions without ultracentrifugation (Figs. 4(a) and 4(c)) were very large, the solutions used were diluted at 1:7 with $\mathrm{D}_{2} \mathrm{O}$. The absorptions in the first and second interband transitions for semiconducting SWNTs $\left(\mathrm{S}_{11}: 900-1300 \mathrm{~nm}, \mathrm{~S}_{22}: 500-800 \mathrm{~nm}\right)$ were observed in all solutions, indicating that the SWNTs were well dispersed in $\mathrm{D}_{2} \mathrm{O}$ using the bath sonicator. Before centrifugation, the spectra showed broadened peaks and a high $\pi$-plasmon baseline level. Well-sharpened peaks were obtained after ultracentrifugation owing to the removal of large SWNT bundles and impurities in the solution, suggesting that individual SWNTs were well dispersed. It is supposed that the number of the dispersed SWNTs in the SDBS solution is more than that in the TP solution because the peak intensity of the SDBS solution is higher at the same surfactant weight. This result corresponds to the apparent darkness of these solutions (Fig. 3(b) and 3(d)). 


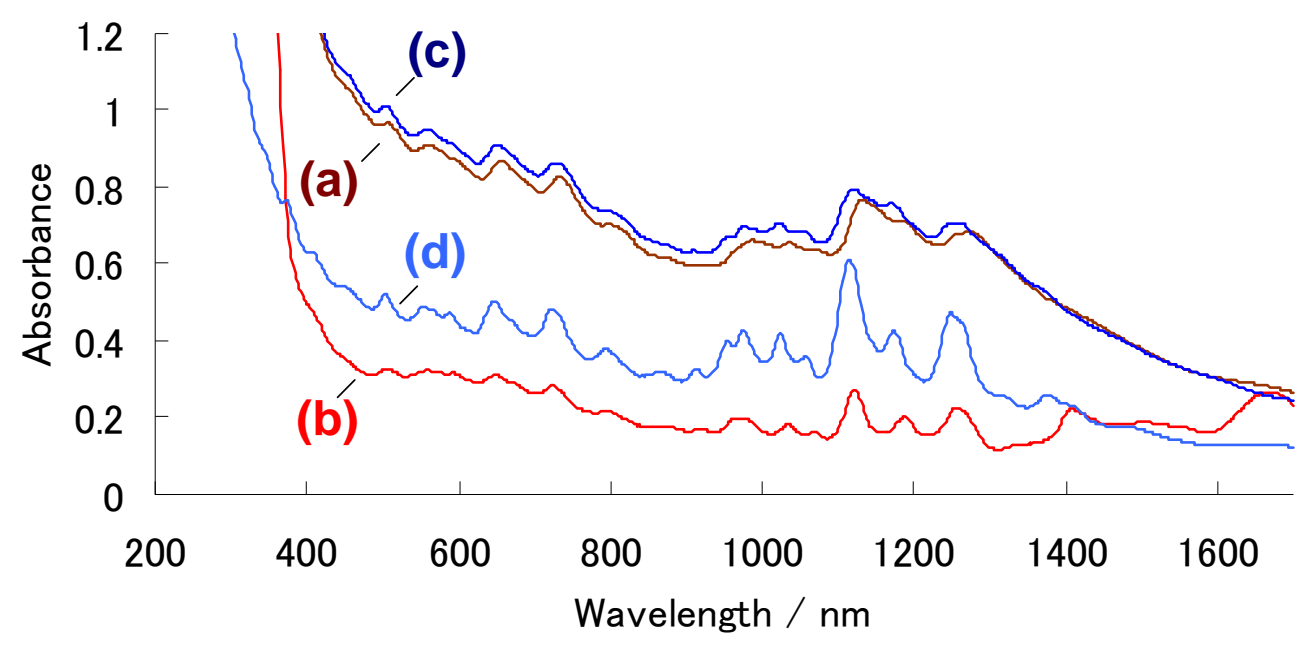

Fig. 4. UV-vis-NIR spectra of a $\mathrm{D}_{2} \mathrm{O}$ dispersion of SWNTs with (a) TP, (b) TP after ultracentrifugation, (c) SDBS, and (d) SDBS after ultracentrifugation.

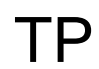

(a)

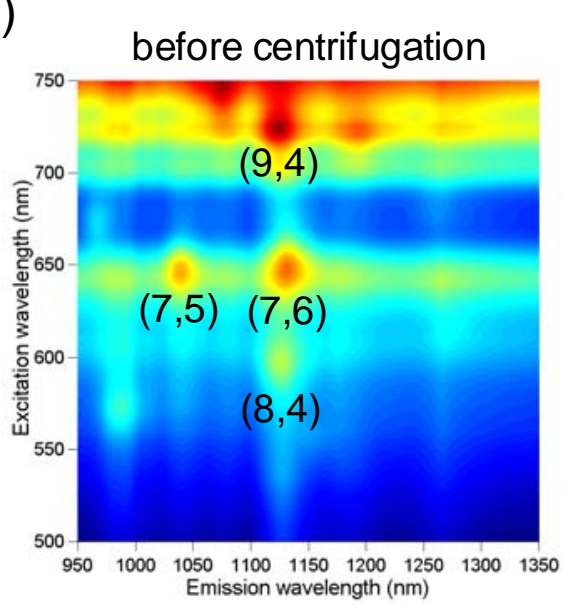

(b)
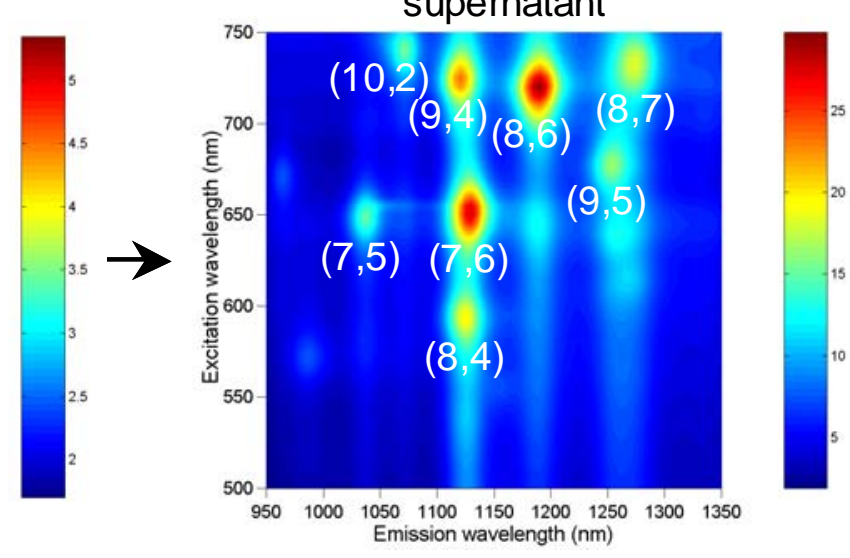

\section{SDBS}

(C)

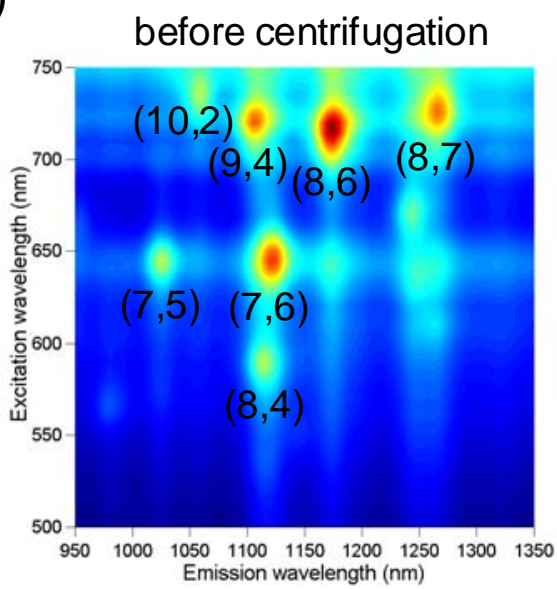

(d)
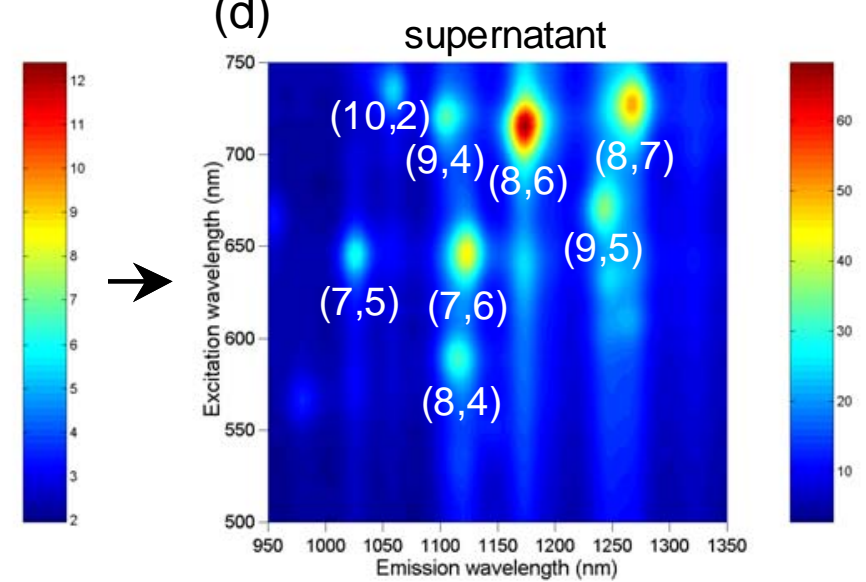

Fig. 5. Photoluminescence maps of $\mathrm{D}_{2} \mathrm{O}$ dispersions of SWNTs with (a) TP, (b) TP after ultracentrifugation, (c) SDBS, and (d) SDBS after ultracentrifugation. 
Furthermore, we measured the PL from individual SWNTs in these solutions for the identification of the sample's $(n, m)$ distribution. PL maps of $\mathrm{D}_{2} \mathrm{O}$ dispersions of SWNTs with (a) TP, (b) TP after ultracentrifugation, (c) SDBS, and (d) SDBS after ultracentrifugation are shown in Fig. 5. Surprisingly, a strong PL from individual SWNTs was observed in these dispersed solutions without ultracentrifugation. From these results, we confirmed the excellent dispersion ability of TP. The brightened regions around the excitation wavelengths of 650 and $800 \mathrm{~nm}$ in Figs. 5(a) and 5(c), were due to the energy transfer between SWNTS in a bundle and due to the low signal-to-noise $(\mathrm{S} / \mathrm{N})$ ratio derived from the low excitation power of the Xe lamp around these wavelengths. The assignment of each chiral index of SWNT is possible without ultracentrifugation. The major PL peaks in the sample prepared with TP were assigned as $(7,5),(8,4)(7,6)$ and $(9,4)$. The emission wavelength of three PL peaks $((9,4),(7,6)$, and $(8,4))$ was around $1150 \mathrm{~nm}$, which corresponds to the strong absorption in the UV-vis-NIR spectra shown in Fig. 4(a). After ultracentrifugation, the PL intensity increased with the suppression of the energy transfer due to the removal of large bundles of SWNTs. The new PL peaks were observed in the sample after ultracentrifugation. Low-intensity PL peaks were observed and assigned as $(9,5)$ and $(8,7)$, which correspond to the large peak absorption at $1250 \mathrm{~nm}$ in Fig. 4(b). Moreover, these peaks assigned as $(10,2)$ and $(8,6)$ were clearly detected. The assigned signals in the PL maps of the TP and SDBS solutions were similar. The $(8,6)$, $(8,7)$ and $(10,2)$ peaks, which were not assigned in the TP solution before ultracentrifugation, were newly assigned in the SDBS solution. The PL intensity ratios of peaks in the TP solution were different from those in the SDBS solution. The PL map of TP-dispersed SWNTs in the $\mathrm{D}_{2} \mathrm{O}$ solution would indicate several strong PL peaks over a wider an $(n, m)$ range than that in the SDBS solution. This different PL pattern indicates different dispersed individual SWNTs in these two surfactants. The structure of TP is very unique in that it is composed of a planar triphenylene group and six undecanoic acids. Therefore, the dispersion mechanism with TP is expected to be different from that with SDBS. Further studies for the clarification of the dispersion mechanism with TP are currently under way in our laboratory.

\section{Conclusions}

In summary, we successfully dispersed individual SWNTs using surfactants in $\mathrm{D}_{2} \mathrm{O}$ solution using a common bath sonicator by a stepwise addition procedure (Fig. 2(a)). The dispersed solution shows clear photoluminescence without ultracentrifugation, and a slight difference between samples prepared with TP and SDBS. A low-power bath sonicator is suitable for non-destructive and long SWNT dispersion because of mild dispersion conditions. Furthermore, the electrical properties of the dispersed SWNTs with TP are interesting because of the triphenylene skeleton of

TP adsorbed to the SWNT surface. ${ }^{20,21)}$ The individually dispersed SWNTs prepared by the stepwise addition procedure could be useful for various applications.

\section{Acknowledgements}

We thank Dr. Robert H. Hauge (Rice University) for kindly supplying purified HiPco SWNTs and Dr. Erik Einarsson (The University of Tokyo) for reviewing the manuscript. This work was supported by grants from the Ministry of Education, Culture, Sports, Science and Technology of Japan and the Takeda Science Foundation. 


\section{References}

1) M. S. Dresselahus and P. C. Eklund: Adv. Phys. 49 (2000) 705.

2) M. J. O’Connell, S. M. Bachilo, C. B. Huffman, V. C. Moore, M. S. Strano, E. H. Haroz, K. L. Rialon, P. J. Boul,W. H. Noon, C. Kittrell, J. Ma, R. H. Hauge, R. B. Weisman, and R. E. Smalley: Science 297 (2002) 593.

3) Y. Lin, S. Taylor, H. Li, K. A. S. Fernando, L. Qu, W. Wang, L. Gu, B. Zhou, and Y.-P. Sun: J. Mater. Chem. 14 (2004) 527.

4) V. C. Moore, M. S. Strano, E. H. Haroz, R. H. Hauge, and R. E. Smalley: Nano Lett. 3 (2003) 1379.

5) A. Ishibashi and N. Nakashima: Chem. Eur. J. 12 (2006) 7595.

6) H. Li, B. Zhou,Y. Lin,L. Gu, W. Wang,K. A. S. Fernando, S. Kumar, L. F. Allard, and Y.-P. Sun: J. Am. Chem. Soc. 126 (2004) 1014.

7) Y. Tomonari, H. Murakami, and N. Nakashima: Chem. Eur. J. 12 (2006) 4027.

8) G. Nakamura, K. Narimatsu, Y. Niidome, and N. Nakashima: Chem. Lett. 36 (2007) 1140.

9) N. Nakashima, S. Okuzono, H. Murakami, T. Nakai, and K. Yoshizawa: Chem. Lett. 32 (2003) 456.

10) M. Zheng, A. Jagota, E. D. Semke, B. A. Diner, R. S. Mclean, S. R. Lustig, R. E. Richardson, and N. G. Tassi: Nat. Mater. 2 (2003) 338.

11) A. O.-Acevedo, H. Xie, V. Zorbas, W. M. Sampson, A. B. Dalton, R. H. Baughman, R. K. Draper, I. H. Musselman, and G. R. Dieckmann: J. Am. Chem. Soc. 127 (2005) 9512.

12) T. Takahashi, C. R. Luculescu, K. Uchida, T. Ishii, and H. Yajima: Chem. Lett. 34 (2005)1516.

13) N. Minami, Y. Kim, K. Miyashita, S. Kazaoui, and B. Nalini: Appl. Phys. Lett. 88 (2006) 093123.

14) M. Zheng, A. Jagota, M. S. Strano, A. P. Santos, P. Barone, S. G. Chou, B. A. Diner, M. S. Dresselhaus, R. S. Mclean, G. B. Onoa, G. G. Samsonidze, E. D. Semke, M. Usrey, and D. J. Walls: Science 302 (2003) 1545.

15) R. Krupke, F. Hennrich, H. V. Löhneysen, and M. M. Kappes: Science 301 (2003) 344.

16) M. S. Arnold, A. A. Green, J. F. Hulvat, S. I. Stupp, and M. C. Hersam: Nat. Nanotechnol. 1 (2006) 60.

17) M. F. Islam, E. Rojas, D. M. Bergey, A. T. Johnson, and A. G. Yodh: Nano Lett. 3 (2003) 269.

18) J. I. Paredes and M. Burghard: Langmuir 20 (2004) 5149.

19) S. M. Bachilo, M. S. Strano, C. Kittrell, R. H. Hauge, R. E. Smalley, and R. B. Weisman: Science 298 (2002) 2361.

20) J. Motoyanagi, T. Fukushima, and T. Aida: Chem. Commun. (2005) 101.

21) Y. Yamamoto, T. Fukushima, Y. Suna, N. Ishii, A. Saeki, S. Seki, S. Tagawa, M. Taniguchi, T. Kawai, and T. Aida: Science 314 (2006) 1761. 